\title{
OPTIMAL RATINGS AND MARKET OUTCOMES
}

\author{
Hugo Hopenhayn \\ Maryam Saeedi \\ Working Paper 26221 \\ http://www.nber.org/papers/w26221
NATIONAL BUREAU OF ECONOMIC RESEARCH
1050 Massachusetts Avenue
Cambridge, MA 02138
September 2019

We acknowledge support of NSF grant \#1757134. The views expressed herein are those of the authors and do not necessarily reflect the views of the National Bureau of Economic Research.

NBER working papers are circulated for discussion and comment purposes. They have not been peer-reviewed or been subject to the review by the NBER Board of Directors that accompanies official NBER publications.

(C) 2019 by Hugo Hopenhayn and Maryam Saeedi. All rights reserved. Short sections of text, not to exceed two paragraphs, may be quoted without explicit permission provided that full credit, including $(\odot$ notice, is given to the source. 
Optimal Ratings and Market Outcomes

Hugo Hopenhayn and Maryam Saeedi

NBER Working Paper No. 26221

September 2019

JEL No. D21,D47,D60,D82,L11

\begin{abstract}
$\underline{\text { ABSTRACT }}$
This paper considers the design of an optimal rating system, in a market with adverse selection. We address two critical questions about rating design: First, given a number of categories, what are the criteria for setting the boundaries between them? Second, what are the gains from increasing the number of categories? A rating system helps reallocate sales from lower- to higherquality producers, thus mitigating the problem of adverse selection. We focus on two main sources of market heterogeneity that determine the extent and effect of this reallocation: the distribution of firm qualities and the responsiveness of sellers' supply to prices. We provide a simple characterization for the optimal rating system as the solution to a standard k-means clustering problem, and discuss its connection to supply elasticity and the skewness of firm qualities. Our results show that a simple two-tier rating can achieve a large share of full information surplus. Additionally, we characterize the conflicting interests of consumers and producers in the design of a rating system.
\end{abstract}

\author{
Hugo Hopenhayn \\ UCLA \\ Department of Economics \\ Los Angeles, CA 90095 \\ and NBER \\ hopen@econ.ucla.edu \\ Maryam Saeedi \\ Tepper School of Business \\ Carnegie Mellon University \\ 5000 Forbes Ave. \\ Pittsburgh, PA 15213 \\ msaeedi@andrew.cmu.edu
}




\section{Introduction}

Certification ratings are widely used in markets with adverse selection. While relevant for any market with asymmetric information (e.g., hygiene ratings for restaurants or doctors' performance ratings), rating design is a key consideration for the overall performance of the ever more popular online trading platforms, where transactions are decentralized and rarely repeated. Despite the importance of rating mechanisms, little is known about their optimal design and how it might depend on the characteristics of the market, such as supply, demand, and the distribution of sellers' qualities. This paper sheds light on this question by considering the design of an optimal rating system and how it relates to the characteristics of the market.

Rating systems usually provide coarse signals of quality to buyers. For example, in California, restaurants are given grades A, B, C, or none based on the score obtained after a hygiene inspection is conducted. Airbnb awards its top-quality hosts the Superhost badge, and eBay's high-quality sellers are classified as Top Rated Sellers. These examples raise two critical questions about rating design: First, given a number of categories, what are the criteria for setting the boundaries between them? In particular, when there are only two categories, how stringent should the standards be to certify a seller is high quality? Second, what are the gains from increasing the number of categories? These choices affect the total market size, the market shares of different types of sellers, and, as a result, the quality distribution of the goods consumed, as supported by recent empirical evidence. ${ }^{1}$ Consequently, a key to rating design is evaluating how these choices impact market performance as measured by consumer and producer surplus. This paper develops a tractable framework to address these questions and provides specific guidelines for the optimal design for different market structures.

A rating system helps reallocate sales from lower- to higher-quality producers, thus mitigating the problem of adverse selection. Our analysis focuses on two main sources of market heterogeneity that are critical to determine the extent and effect of this reallocation: the distribution of firm qualities and the responsiveness of sellers' supply to prices. Intuitively, the heterogeneity and skeweness of seller quality affect the spread of prices across ratings, while the responsiveness of supply determines the resulting reallocation of output across these categories. To our best knowledge, this is the first paper that considers systematically the interaction of these two factors and their impact on optimal rating design.

The following is a preview of our results. We first show that while better information always increases total surplus, its effect on consumers depends on the elasticity of supply. Second,

\footnotetext{
${ }^{1}$ Hui et al. (2018) examine the effect of an increase in the requirements to become a badged seller on eBay. They find that this increase leads to a higher market share of high-quality sellers while decreasing the sales of sellers in the medium range of quality.
} 
we show that the optimal information structure with finite ratings can be found in the set of threshold partitions, which are related to the solution of a standard k-means clustering problem. Right skewness in the distribution of qualities or more elastic supply implies higher thresholds and a lower share of certified sellers. Third, we identify theoretical bounds for the performance of a simple two-tier rating system and provide numerical computations showing it can achieve 46-77\% of full information surplus for some standard classes of distributions.

Our baseline model considers a competitive market with a large set of buyers and sellers. ${ }^{2}$ Firms are endowed with different levels of quality, which is the only source of product differentiation. ${ }^{3}$ Production technology is the same for all firms. All buyers have the same preference for quality but are heterogeneous in their value of the outside option. As explained below, the information structure partitions sellers into different levels of expected quality. An equilibrium is a set of prices as a function of expected quality and quantities supplied by firms such that markets clear. Our model exhibits two features that are common to adverse selection settings that are affected by the degree of asymmetric information. First, low-quality sellers benefit from being pooled with high-quality ones, while adversely affecting them. Second, high-quality sales are crowded out by low-quality ones.

The information structure shared by all buyers follows the setting described in Ganuza and Penalva (2010) and Gentzkow and Kamenica (2016). A common prior over firm qualities and a signal structure determine the distribution of expected posterior firm qualities. As an example, if we consider the case of finite rating systems as those described above, the posterior is given by a discrete distribution with point masses at the conditional mean qualities associated to each rating. This setting provides a natural ordering of the quality of information, where better information is associated to a mean-preserving spread of the distribution of expected values.

As a preliminary step we analyze the effect of improving information on equilibrium outcomes. As a result of improved information, prices become more strongly associated with true seller quality and thus more disperse. Demand is reallocated from lower- to higher-quality firms, which has a positive effect on the average quality of goods consumed and total surplus. However, its effect on total market size and consumer surplus is ambiguous and depends on the properties of the supply function. When supply is convex, the higher spread in prices results in an increase in total output and higher consumer surplus. The opposite occurs when the supply

\footnotetext{
${ }^{2}$ We also consider the case of Cournot competition with constant marginal costs and show the results are the same as those for perfect competition with linear supply.

${ }^{3}$ While moral hazard might be a critical consideration in some markets, in others adverse selection might play a more critical role, as suggested by an empirical study on eBay (see Hui et al. (2017)). Optimal rating design with moral hazard and adverse selection is considered in Saeedi and Shourideh (2019) in a simplified market environment.
} 
is concave. In this case, any information provided by the market designer lowers consumer surplus. We also show that profits increase with information quality when the supply function is concave, while the effect is ambiguous otherwise.

We then turn to the question of optimal rating design. While we focus primarily on total surplus as a criterion, we also consider the effect on consumer and producer surplus. Since total surplus increases with better information, an unconstrained designer would prefer to release all information. However, in practice, many platforms use a limited number of categories, as in the examples discussed above, as well as certifiers such as bond rating agencies. ${ }^{4}$ To provide guidance in the design of such rating systems, we consider the problem of optimal rating design when the designer is constrained to choose a fixed number of categories $N$.

We show that the optimal information structure with finite ratings can be found in the set of threshold partitions, i.e., those that partition sellers into ordered, disjoint, and connected intervals of quality in a monotone fashion. This reduces the problem of optimal ratings to the determination of the corresponding set of optimal thresholds, which are defined by an intuitive criterion. Consider a marginal firm with quality at the threshold between two adjacent intervals. For this threshold to be optimal, the planner should be indifferent between placing this marginal firm in the lower or upper interval. This decision ultimately affects the demand faced by this firm, and thus its total output. The benefit of the increased output is the extra value generated by the additional sales, which at the optimum should be equated to the extra cost of production. This suggests that one of the key determinants of this trade-off is the supply behavior of firms, in particular, the curvature of the supply function.

The role of curvature can be conveniently illustrated comparing the two polar cases of perfectly inelastic and perfectly elastic supply. When supply is perfectly inelastic (i.e., producers can produce either zero or one unit or equally face a constant marginal cost up to a fixed capacity), quality ratings cannot reallocate output to higher-quality producers, except in the extreme case where there is no production in the absence of information. However, ratings can potentially serve to filter out the very low-quality producers that no consumer would buy from at a positive price, so the optimal threshold will be at this low end. At the other extreme, when all firms face a constant marginal cost, only the highest-quality firm should serve the market, so the optimal threshold would be at the other end. More generally, we find that the optimal thresholds in the case of a convex (resp. concave) supply function are pointwise higher (resp. lower) than those in the linear case. As an example, in the case of a simple certification rating with two groups, more elastic supply leads to a higher threshold and lower share of certified sellers.

\footnotetext{
${ }^{4}$ This can be justified by practical reasons, to reduce the cost of acquiring information or to keep the rating system simple and transparent.
} 
We find a simple characterization for the optimal thresholds in the case of linear supply, which provides a useful benchmark. These optimal thresholds are the solution to a standard clustering problem that involves only information regarding the distribution of qualities. This clustering problem can be solved by the $k$-means algorithm as introduced by MacQueen et al. (1967) and used extensively in machine learning and statistics. Our results thus provide a straightforward and easy-to-compute method for the design of rating systems.

We then consider the thresholds that maximize consumer and producer surplus. To highlight the conflict of interest between consumers and producers, we have already noted that in the case of concave supply functions, consumer surplus decreases while profits increase with better information. More generally, the thresholds that maximize total surplus are in between those that maximize producer and consumer surplus. When supply is linear, consumer surplus does not change with the choice of ratings and the optimal thresholds for total surplus also maximize total profits, so the ranking of optimal threshold is indeterminate. On the sides of this knife-edge case, we provide sufficient conditions for the ordering of optimal thresholds. In particular, we find a clear ranking for the class of homogeneous supply functions that are widely used in applications. When the degree is greater than one (convex supply) producers prefer higher thresholds, i.e., more stringent standards, while consumers prefer lower ones. The reverse occurs when the degree is less than one.

Next, we relate the optimal thresholds to the distribution of firm qualities in the linear supply case. In the case of a single threshold, the optimal share of certified sellers relates to the skewness of the distribution: when it is right (resp. left) skewed, the share is above (resp. below) one half. We also show that if the distribution of qualities is more skewed to the right then the optimal percentiles of thresholds defined for the first will be higher. In particular, for a two-tier certification rating, the share of certified firms should decrease with the degree of right-skewness. Optimal thresholds are considerably high for some distributions; as an example, when the distribution of qualities is exponential with any hazard rate, only $20 \%$ of sellers should be certified.

Regarding the performance of ratings, we show that a one-threshold partition closes at least half of the surplus gap between no information and full information for quality distributions with log-concave density. In our numerical computations we find that it closes from $46 \%$ to nearly $77 \%$ of the gap, depending on the underlying distribution of qualities. The gains diminish rapidly as the number of thresholds increases. Even though our results show that the higher the number of certification tiers, the higher the total surplus, the market designer should weigh in the cost of having a more complex information structure against the diminishing return of having more tiers. As an illustration of our methodology, we solve for the optimal threshold in a two-tier partition using data from eBay. 
The last part of the paper examines a series of extensions. Our first extension considers a demand system where agents have heterogeneous preference for quality, and firms have inelastic supply. While by construction, improvements in information do not increase total quantity, they contribute to welfare by increasing the correlation between average firm quality and consumer preference for quality. The optimal threshold is defined by a slightly modified formula that weighs differences in the firm's quality gap in each interval by the respective gap in consumers' preferences. As a result, skewness in consumers' preferences for quality has similar implications to the ones observed for skewness in producers' quality.

Second, we consider Cournot competition and show that all the results obtained under perfect competition for the linear supply case apply to this setting. In our final extension, we add entry to the baseline model. Improvements in information induce entry, as a result of the increase in profits. Entry reduces prices, increases market size, and, thus, consumer surplus. In the extreme case where all potential entrants are ex-ante identical, drawing their qualities after entry, all gains from improved information accrue to consumers.

\section{Related Literature}

Our paper is related to two strands of the literature: first, the papers considering the impact of information disclosure on consumer and producer surplus; second, those concerning the determinants of ratings systems as well as their performance. ${ }^{5}$

In regards to the impact of information disclosure, most papers consider the case where there is a single seller/auctioneer and multiple buyers, as opposed to multiple agents on both sides. Similar to our results, Schlee (1996) shows that information can hurt consumers when the cost function is sufficiently convex. Bergemann et al. (2015) consider the impact of information in third degree price discrimination. They show that any distribution of surplus that is between the ones achieved by optimal pricing with none and full information can be attained with some information structure. Bergemann and Pesendorfer (2007) show that in a private value setting, bidders can be worse off with better information even though total surplus increases. Board (2009) shows that this result depends on the number of bidders. Hoppe et al. (2011) consider a matching problem where for some distribution of types, consumers can be worse off with better information. In our paper we show that better information always increases total surplus, but might decrease consumer or producer surplus depending on properties of the supply function.

There is a large literature on certification and quality disclosure. Dranove and Jin (2010) provide an excellent survey of the earlier papers. One of the first theoretical papers is Lizzeri

\footnotetext{
${ }^{5}$ Our paper focuses on a setting where uncertainty is about seller quality and information is provided to consumers. There is a growing literature that focuses on the reverse channel where an intermediary transmits information about buyers to sellers. For a survey see Bergemann and Bonatti (2019).
} 
(1999), that considers the effect of competition among certifiers. The author finds that while a monopoly certifier chooses to provide coarse information with a single and low threshold, competition among certifiers can lead to full information. Ostrovsky and Schwarz (2010) consider equilibrium information structures and show that colleges maximize student job prospects by pooling weaker and stronger students. De Marzo, Kremer, and Skrzypacz (2018) consider a Bayesian game where agents choose the informativeness of testing, but can withhold bad results. The equilibrium test minimizes the expected price upon nondisclosure over all possible tests and disclosure strategies and results in a threshold that is below the optimal one. In contrast, to these papers, in our setting, the preferred thresholds of sellers can be below or above the optimal ones depending on properties of the supply function.

A growing literature considers the impact of moral hazard and experimentation incentives on ratings design. The general finding is that coarse ratings can contribute to provide better incentives. Harbaugh and Rasmusen (2018) consider a case where the certifier discloses limited information in order to incentivize participation. Examples of papers related to moral hazard and limited information/memory are Hörner and Lambert (2016), Liu and Skrzypacz (2014), Ekmekci (2011), and Bhaskar et al. (2017). Examples of papers where limited information encourages experimentation are Kovbasyuk and Spagnolo (2017), Che and Hörner (2018), Kremer et al. (2014) and Vellodi (2019). The latter also considers information design and its effect on entry and selection of firms.

Coarse ratings have also been justified in the literature by their simplicity and overall performance. Wilson (1989) shows that losses relative to full information are of order $1 / n^{2}$ for a partition with $n$ classes. This is consistent with our computed bounds in Section 5 . Our theoretical bound on the gains from a two-tier certification builds on similar bounds found by McAfee (2002) and also relate to those in Hoppe et al. (2011).

Information disclosure is the focus of the literature on Bayesian persuasion, where an informed sender chooses an information structure to influence the behavior of a receiver. This literature generally finds coarse information provision as a result. Dworczak and Martini, (2018) provide conditions on payoffs so that interval partitions are the optimal information structure. In contrast to most of this literature, where a single receiver takes an action, in our setting the outcome is the result of the equilibrium choices of multiple agents. ${ }^{6}$ This distinction

\footnotetext{
${ }^{6}$ While other papers have studied settings with multiple receivers (see surveys in Kamenica (2018); Bergemann and Morris (2017)), the analysis has often been suitable for games where a low-dimensional source of aggregate information is observed by a sender. For example, Bergemann and Morris (2013, 2016) characterize the outcome of all Bayesian persuasion games with multiple receivers. In principle, our problem could be potentially mapped into this framework, with an omniscient sender that observes the quality of a continuum of firms, but it would be impractical to solve it this way. Even for a simple two-player game, Bhaskar et al. (2016) show that computing the optimal public signal is NP-hard.
} 
applies to most of the literature reviewed above, which often ignores market interactions, in contrast to the approach followed in this paper where it is a key consideration. While individual incentives for quality disclosure are important, they seem less relevant for platforms wherein a central authority observes some information and must choose what to reveal to the market participants; this is what we focus on in our analysis.

The most relevant empirical papers related to our theory are Saeedi (2014), Elfenbein et al. (2015), Fan et al. (2013), and Jin and Leslie (2003). Saeedi (2014) studies the value of reputation mechanisms and establishes a positive signaling value for the certification done by eBay. Elfenbein et al. (2015) study the value of certification badges across different markets. They find that certification provides more value when the number of certified sellers is low and when markets are more competitive. Fan et al. (2013) analyze the effect of badges on Taobao.com. They find sellers offer price discounts to move up to the next reputation level. Jin and Leslie (2003) examine the effect of an increase in product quality information to consumers on firms' choices of product quality using data on restaurant hygiene ratings. Our paper also relates to the literature that analyzes the effects of changes in a marketplace feedback mechanisms on price and quality (Hui et al. (2016), Filippas et al. (2018), and Nosko and Tadelis (2015)). ${ }^{7}$

Section 2 describes the model. Section 3 considers the effect of improved information on market outcomes. Section 4 derives the conditions that characterize optimal thresholds, the comparative statics on supply functions and discusses the implications for consumer and producer surplus. Section 5 provides comparative statics on distribution functions, numerically solves for the optimal thresholds for various distribution functions and considers the application to eBay ratings. Finally, Section 6 studies the extensions to the baseline model.

\section{The Model}

There is a unit mass of firms with qualities $z$ distributed according to a continuous cumulative distribution function (cdf) $F(z)$. Production technology is the same for all firms and is given by a continuous, strictly increasing, and strictly convex cost function $c(q)$, and, correspondingly, a strictly increasing supply function $s(p)$. On the demand side, there is mass $M$ of consumers who face a discrete choice problem, with preferences

$$
U(z, \theta, p)=z+\theta-p,
$$

\footnotetext{
${ }^{7}$ More generally, this research also fits broadly into the theme of understanding the effect of certification and reputation in e-commerce; representative papers include Cabral and Hortacsu (2010), Wu et al. (2015), Tadelis (2016), Dellarocas et al. (2006), Bajari and Hortacsu (2004), and Chevalier and Mayzlin (2006).
} 
where $z$ is the quality of the good purchased, and $\theta$ is a taste parameter measuring the preference for goods offered in this market vis a vis an outside option and $p$ is the price of the good. The taste parameter $\theta$ is distributed according to a continuous and strictly increasing cdf $\Psi(\theta)$, while the outside good's utility (no purchase) is normalized to zero. ${ }^{8}$ Goods are differentiated only by quality that is equally valued by all consumers. ${ }^{9}$ Given the linearity of the utility function in $z$, the same ordering is obtained for consumption of a good of expected quality $z$. We assume all market participants have the same information about the expected qualities of firms, represented by the distribution function $G(z) \cdot{ }^{10}$ In particular, when considering a finite rating system as in Section 4 , we assume that $G$ is a discrete distribution with point masses at the conditional mean qualities associated to each rating. We will say that a firm has expected quality $z$ if conditional on all signals received, that is the quality expected by consumers.

Given expected quality $z$, equilibrium prices take the form $p(z)=p(0)+z$, where $p(0)$ corresponds to the demand price of a hypothetical good of quality zero. This expression for prices guarantees that all consumers are indifferent between goods with different signal realizations, which is a necessary condition for an equilibrium. Given a baseline price $p(0)$, the marginal consumer's $\theta$ is found by setting $U(0, \theta, p(0))=0$, or simply $\theta(p(0))=p(0)$. All consumers with $\theta \geq p(0)$ will consume some good, so aggregate demand is $Q=M(1-\Psi(p(0)))$. Inverting this function we can define an inverse baseline demand function

$$
P(Q)=\Psi^{-1}(1-Q / M)
$$

On the supply side, each firm with expected quality $z$ chooses its output, $q=s(p(z))$, so aggregate supply $Q=\int s(p(z)) d G(z)$.

Definition. An (interior) equilibrium, given the distribution of expected qualities $G(z)$, is given by prices $p(z)=P(Q)+z$, where total quantity $Q=\int s(p(z)) d G(z)$.

Figure 1 shows graphically the derivation of an interior equilibrium for the case of a twotier partition, where $L$ represents the group of firms with quality below a threshold $z^{*}$, and $H$ those above. ${ }^{11}$ Denote $z_{L}$ (resp. $z_{H}$ ) to be the average quality of sellers in the $L$ group (resp. $H$ group). The two curves depict the demand curve for the goods in the $L$ and $H$ segment, respectively. Since all consumers value quality identically, the price difference

\footnotetext{
${ }^{8}$ Alternatively, one can consider $-\theta$ to be the value of the outside good to consumers.

${ }^{9}$ In Section 6.1, we consider a case where consumers are heterogenous with respect to their taste for quality.

${ }^{10}$ This representation of the information structure is consistent with the approach followed in Ganuza and Penalva (2010) and Gentzkow and Kamenica (2016). Given a common prior $F\left(z_{0}\right)$ over firm qualities and a signal structure $\pi$, we can let $G(z)$ be the distribution of expected posterior firm quality.

${ }^{11}$ Alternatively, this can be interpreted as a case of having two type of sellers with two levels of qualities.
} 


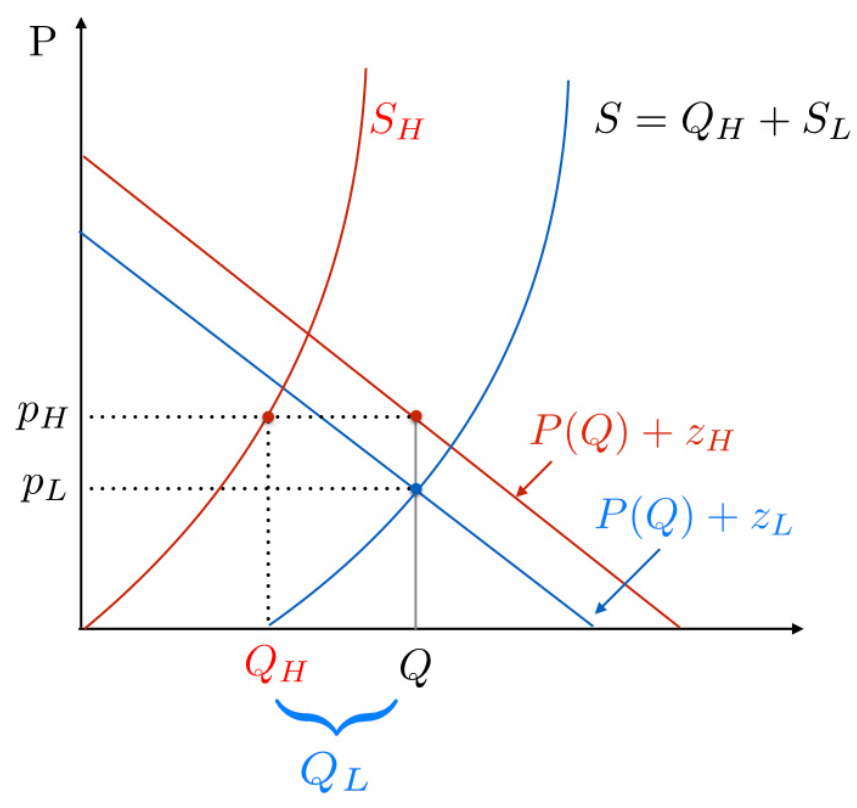

Figure 1: Equilibrium

$p_{H}-p_{L}=z_{H}-z_{L}$, i.e., the difference between the two respective average qualities. The first upward sloping curve is the $H$ group's supply function: $S_{H}=\left(1-F\left(z^{*}\right)\right) s\left(p_{H}\right)$. The second one is the supply function of the $L$ firms' segment $S_{L}=F\left(z^{*}\right) s\left(p_{L}\right)$ displaced to the right by the equilibrium quantity $Q_{H}$. The marginal consumer $Q$ is the one that is indifferent between consuming either of these goods or none. At the equilibrium prices, $Q$ is also the total market supply of both goods.

To prove the existence of an interior equilibrium, we make the following assumption.

Assumption 1. There exists $\underline{\theta}$ in the support of $\Psi$ such that

$$
M>\int s(\underline{\theta}+z) d G(z)
$$

for all distributions $G$ such that $F$ is a mean preserving spread of $G$.

This assumption rules out the possibility that all consumers make purchases in this market. ${ }^{12}$ While a corner equilibrium, if it exists, is also unique, we rule this out as a matter of convenience.

\footnotetext{
${ }^{12}$ As explained below, the assumption spans the set of all possible information structures.
} 
Lemma 1. Under Assumption 1, there exists a unique interior equilibrium for all expected quality distributions $G$ such that $F$ is a mean preserving spread of $G$.

Proof. Given that the cdf $\Psi$ is strictly increasing and continuous, the function $P(Q)$ is strictly decreasing and continuous. Define function $f(Q)=\int s(P(Q)+z) d G(z)$. This is strictly decreasing and continuous. It follows immediately that $f(0)>0$. By assumption $1, f(M)<$ $M$ since $P(M) \leq \underline{\theta}$. Hence, there exists a unique fixed point $Q^{*}$ for this mapping.

\section{Improved Information}

This section considers the effect of improved information for general signal structures. As in Ganuza and Penalva (2010), the quality of information can be ordered by the dispersion of beliefs as follows. The distribution of expected qualities $\tilde{G}$ is more informative than distribution $G$ if it is a mean preserving spread of $G$. We will refer to this ordering as better information. As the maximal signal structure corresponds to perfect information, the class of all information structures can be represented by all garblings of $F$, i.e., all distributions $G$ such that $F$ is a mean preserving spread of $G .{ }^{13}$

\section{Market Size and Consumer Surplus}

We consider first the effect of information precision on market size measured by the equilibrium quantity $Q$. For fixed quantity $Q$, better information implies a mean-preserving spread of prices. In the case of linear supply functions, this does not affect aggregate supply, so equilibrium quantity remains unchanged. In the case of convex supply functions, aggregate supply will exceed the original quantity $Q$, so aggregate output must increase. For similar reasons, in the case of concave supply functions, aggregate output decreases with the quality of information.

We show that consumer surplus can be measured by the area under the baseline demand function and over $P(Q)$, up to quantity $Q$. Consider a consumer of type $\theta$ who buys a good of quality $z$. Equilibrium price for this good $p(z)=P(Q)+z$, so this consumer's net utility

\footnotetext{
${ }^{13}$ This corresponds to the ordering of integral precision of signal structures defined in Ganuza and Penalva (2010). Starting from a prior $F_{0}$, signal structure $\tilde{t}$ is more integral precise than signal $t$ if the induced distribution of expected qualities $G(z)$ generated by $\tilde{t}$ is a mean-preserving spread of the one generated by $t$. In general, integral precision ordering is weaker than the likelihood ratio and other related orderings considered in the literature (see Ganuza and Penalva (2010) for references.)
} 
is $\theta-P(Q)$. It follows that total consumer surplus is

$$
\int_{P(Q)}(\theta-P(Q)) d \Psi(\theta)=\int_{0}^{Q}(P(x)-P(Q)) d x
$$

where the last equality follows from the change of variables $x=M(1-\Psi(\theta))$ and our definition of $P(Q)$ given by equation (1). As a result, total consumer surplus will move in the same direction as total quantity, so it will increase (resp. decrease) with information precision if the supply function is convex (resp. concave). The following proposition summarizes these results.

Proposition 1. Suppose signal $t_{1}$ is more precise than $t_{2}$. If in addition the supply function is convex (resp. concave), then total output $Q_{1} \geq Q_{2}$ (resp. $Q_{2} \leq Q_{1}$ ) and consumer surplus increase (resp. decrease). This further implies that when the supply function is linear, total output is independent of information.

Proof. Suppose the supply function $S(p)$ is convex and, by way of contradiction, $Q_{1}<Q_{2}$. Let $p_{1}(z)=P\left(Q_{1}\right)+z$ denote the equilibrium price for a good of expected quality $z$ and define similarly $p_{2}(z)$. It follows immediately that $p_{1}(z)>p_{2}(z)$ since $P$ is strictly decreasing. Letting $G_{1}$ and $G_{2}$ denote the distribution of expected qualities under signal structures $t_{1}$ and $t_{2}$, respectively. By definition of integral precision, it follows that $G_{1}$ second order stochastically dominates $G_{2}$, so

$$
\begin{aligned}
Q_{1} & =\int S\left(p_{1}(z)\right) d G_{1}(z) \geq S\left(p_{2}(z)\right) d G_{1}(z) \\
& \geq \int S\left(p_{2}(z)\right) d G_{2}(z)=Q_{2}
\end{aligned}
$$

where the second inequality follows from convexity of $S(p)$. The above contradicts the original hypothesis, proving that $Q_{1} \geq Q_{2}$. The proof is similar for concave $S(p)$.

In particular, note that for the case of concave supply functions, consumers are better off with no signal provision. There are some related results in the literature, though in different settings. Schlee (1996) considers a single product monopoly seller in a vertically differentiated market. The quality of the good offered is exogenous and privately observed by the monopolist, who must choose the informativeness of a signal to be provided to consumers before observing the quality realization. It is shown that if the cost function is sufficiently convex, consumers are worse off ex-ante with a more informative signal. Hoppe et al. (2011) consider a matching problem and show that under some conditions on the distribution of types, one of the sides 
(e.g., consumers) can be worse off by having a more precise information structure regarding the type of the other side.

\section{Total Surplus and Profits}

In this part, we show that improved information always increases total surplus. The intuition is as follows. First, we show that a social planner, subject to the the same information structure, cannot improve on the competitive equilibrium allocation, which is thus optimal. Secondly, equipped with better information a social planner can always increase total surplus.

Proposition 2. A better information system results in higher total surplus.

Proof. First, we show that there exists a correspondence between competitive equilibria and allocations that maximize total surplus. Given a distribution of mean qualities $G(z)$, the problem of maximizing total surplus solves:

$$
S=\max _{q_{k}} \int_{0}^{Q} P(x) d x+\int[z q(z)-C(q(z))] d G(z)
$$

subject to

$$
Q=\int q(z) d G(z)
$$

The first order conditions for the choice of $q(z)$ is:

$$
z-C^{\prime}(q(z))+\lambda=0
$$

and this holds for all points in the support of $G$, where the Lagrange multiplier of the constraint, $\lambda=P(Q)$. Substituting in (2) and letting $p(z)=P(Q)+z$, implies $p(z)=C^{\prime}(q(z))$ which is the condition defining the profit maximizing output $q(z)$ in the competitive equilibrium. Hence the allocation $q(z)$ together with the prices $p(z)$ are the ones that correspond to the unique competitive equilibrium.

Suppose the distribution of expected qualities $\tilde{G}$ corresponds to a better information system than $G$, so it is a mean preserving spread of $G$. Following the characterization in Rothschild and Stiglitz (1970), there exists a garbling of signals that generates $G$ from $\tilde{G}$. This means that a social planner could ignore the additional information contained in $\tilde{G}$ and reproduce the quantity-weighted distribution of average qualities corresponding to the optimal allocation under $G$ and thus the same value. Since this is not optimal, because the competitive equilib-

rium given $\tilde{G}$ (which is optimal) differs from that under $G$, it follows that total value increases with signal precision. 
In particular, the above proposition implies that average profits must rise when consumer surplus does not increase. Following Proposition 1, this will be the case when supply functions are concave or linear. On the other hand, it is easy to construct examples with convex supply functions where the opposite occurs.

\section{Optimal Ratings}

This section considers the question of optimal information design. While we focus on total surplus as a criterion, we also consider the effect on consumer and producer surplus. As stated by Proposition 2, better information is preferred to worse, so an unconstrained designer would prefer to release all information. However, in practice, many platforms use a limited number of categories. For example, in the case of Yelp, the partition involves five stars, including the possibility of half-stars. In the case of eBay, the partition includes two groups: the badged and unbadged. In the case of California restaurants, the partition involves three elements: A, $\mathrm{B}$, and $\mathrm{C}$. To provide guidance in the design of such rating systems, we consider the optimal information structure design subject to a fixed set of categories $N$.

To simplify our analysis, we assume that consumers have no information other than that provided by the certifier. The timing is as follows: 1) Certifier observes some signals for each firm that are correlated with their quality; 2) Certifier assigns a rating to each firm and makes it common knowledge to all participants in the market. Based on these ratings all market participants can infer the average quality of sellers, thus sharing a common posterior with support at the corresponding $N$ conditional quality means. ${ }^{14}$ 3) Market equilibrium outcomes are determined for this distribution of expected qualities. Following our earlier discussion on information structures, the certifier's information can be summarized by a distribution of posterior mean qualities that, in order to avoid further notation, we denote by $F(z)$. This is the basis on which the certifier classifies firms into rating bins. To simplify the exposition, we refer to the expected value $z$ as the quality of the firm. ${ }^{15}$ We assume $F$ is differentiable on its support with density $f(z)$.

A threshold partition totally orders firms into $N$ quality intervals. As a corollary to Proposition 2, we establish the superiority of threshold partitions. ${ }^{16}$

\footnotetext{
${ }^{14}$ Certifiers usually provide users with guidelines to interpret their ratings or users learn their meaning over time.

${ }^{15}$ The true distribution of qualities plays no role, as any information provision that respects the information of the certifier is a garbling of the certifier's posterior $F$.

${ }^{16}$ Dworczak and Martini (2019) provide conditions under which the optimal signal structure implies a monotone partition in a Bayesian persuasion setting. Our setting is quite different and our result quite straightforward. We can only find a connection in the case of linear supply, where our problem can be mapped into their
} 
Corollary 1. The optimal rating is given by a threshold partition.

Proof. Consider a partition of the set of sellers into sets $S_{1}, \ldots, S_{N}$. Suppose there are two sets $S_{k}, S_{k+1}$ that are not totally ordered in quality. One can substitute $S_{k}$ and $S_{k+1}$ by two new disjoint sets $S_{k}^{\prime}$ and $S_{k+1}^{\prime}$ of equal measures to the original ones that are totally ordered so that $S_{k}^{\prime}<S_{k+1}^{\prime}$, element-wise. This generates a mean-preserving spread of the original distribution of means and thus higher surplus.

Section 4.1 considers the ratings that maximize total surplus and the next section considers consumer and producer surplus.

\subsection{Total Surplus}

We now characterize the threshold partition that maximizes total surplus. Consider a vector of thresholds $\boldsymbol{z}=\left(z_{1} \ldots z_{N-1}\right)$ defining the $N$ partitions $\left\{\left[z_{0}, z_{1}\right],\left[z_{1}, z_{2}\right], \ldots,\left[z_{N-1}, z_{N}\right]\right\}$, where $z_{0}$ and $z_{N}$ are the lower and upper supports of the distribution of qualities $(-\infty$ or $+\infty$ if unbounded), respectively. Let $m\left(z_{k-1}, z_{k}\right), k=1, \ldots, N$ denote the conditional means of quality $z$ in the intervals $\left[z_{k-1}, z_{k}\right]$. For simplicity, we will use the notation $M_{k}=m\left(z_{k-1}, z_{k}\right)$ unless otherwise needed for clarity. The following lemma gives necessary and sufficient conditions for these thresholds to be optimal. Let $Q(\boldsymbol{z})$ denote the unique equilibrium total quantity at the optimal threshold vector, with prices $p_{k}=P(Q(\boldsymbol{z}))+M_{k}$ and quantities $q_{k}=s\left(p_{k}\right)$. Total surplus is given by

$$
W(\boldsymbol{z})=\int_{0}^{Q(\boldsymbol{z})} P(x) d x+\sum_{k=1}^{N}\left[F\left(z_{k}\right)-F\left(z_{k-1}\right)\right]\left[M_{k} q_{k}-c\left(q_{k}\right)\right]
$$

The following lemma gives the necessary conditions for an optimal $\mathrm{z}_{k}$.

Lemma 2. A necessary condition for an optimal threshold $z_{k}$ is that

$$
\left(P(Q)+z_{k}\right)\left(q_{k+1}-q_{k}\right)=c\left(q_{k+1}\right)-c\left(q_{k}\right)
$$

Proof. To totally differentiate equation (3) with respect to $z_{k}$, first note that by the envelope condition, we can ignore the effect on the output choices $q_{1}, \ldots, q_{N}$. In particular, this implies that $\partial Q / \partial z_{k}=f\left(z_{k}\right)\left(q_{k}-q_{k+1}\right)$. Since $M_{k}=\int_{z_{k-1}}^{z_{k}} z d F(z) /\left(F\left(z_{k}\right)-F\left(z_{k-1}\right)\right)$, it follows that

$$
\frac{\partial\left(F\left(z_{k}\right)-F\left(z_{k-1}\right)\right) M_{k}}{\partial z_{k}}=f\left(z_{k}\right) z_{k}, \frac{\partial\left(F\left(z_{k+1}\right)-F\left(z_{k}\right)\right) M_{k+1}}{\partial z_{k}}=-f\left(z_{k}\right) z_{k} .
$$

formulation with the additional constraint of a fixed number of ratings. 
The result now follows by totally differentiating (3) and setting it equal to zero.

Condition (4) has an intuitive interpretation. The left hand side shows the marginal value obtained by increasing the quantity of the marginal firm with quality $z_{k}$, from $q_{k}$ to $q_{k+1}$; this would result from a marginal change in this threshold. The right hand side shows the difference in cost. At an optimum, these two should be equal. While intuitive, all the variables in this equation, quantities and prices, are endogenous to the choice of thresholds, thus limiting the usefulness in characterizing the optimal thresholds. The following Lemma provides a more useful characterization.

Lemma 3. The optimal thresholds satisfy the following condition:

$$
\frac{z_{k}-M_{k}}{M_{k+1}-M_{k}} s\left(p_{k}\right)+\frac{M_{k+1}-z_{k}}{M_{k+1}-M_{k}} s\left(p_{k+1}\right)=\frac{\int_{p_{k}}^{p_{k+1}} s(p) d p}{p_{k+1}-p_{k}},
$$

where $M_{k}$ and $M_{k+1}$ are the conditional mean qualities for the two groups and $p_{k}$ and $p_{k+1}$ the equilibrium prices.

Proof. See Appendix.

Equation (5) equates the expected value of $s(p)$ under two lotteries. The left hand side lottery has weights $\alpha=\left(z_{k}-M_{k}\right) /\left(M_{k+1}-M_{k}\right)$ on price $p_{k}$ and $(1-\alpha)$ on price $p_{k+1}$. The second lottery is uniform between these two extreme prices. When $s$ is linear, it must be the case that $\alpha=1 / 2$ and this implies that

$$
z_{k}-M_{k}=M_{k+1}-z_{k}
$$

When $s$ is convex, $\alpha>1 / 2$ so $z_{k}-M_{k}>M_{k+1}-z_{k}$, so the optimal threshold is above the one defined by equation (6), while the reverse occurs when $s$ is concave. The following lemma summarizes these results.

Lemma 4. If the supply function is linear, the optimal threshold $z_{k}=\left(M_{k}+M_{k+1}\right) / 2$. If the supply function is concave (resp. convex), then $z_{k}$ is lower (resp. higher) than $\left(M_{k}+M_{k+1}\right) / 2$.

While the second part of this lemma gives criteria for local deviations for a single threshold, convex to the right and concave to the left, starting at those obtained for the linear case, it does not imply an ordering of the whole vector of thresholds. The following proposition gives conditions for the total ordering.

Proposition 3. Suppose the quality distribution $F(z)$ has log-concave density. Let $\left(z_{1}^{L}, \ldots, z_{N-1}^{L}\right)$ be the optimal thresholds for the linear case. The optimal vector of thresholds $\left(z_{1}, \ldots, z_{N-1}\right)$ for convex (resp. concave) supply function is pointwise higher (resp. lower) than $\left(z_{1}^{L}, \ldots, z_{N-1}^{L}\right)$. 
Proof. See Appendix.

The formula given by equation (6) gives a simple characterization for the optimal thresholds for the linear supply case that only depends on the distribution of qualities and by the previous proposition provides a lower (resp. upper) bound when the supply function is convex (resp. concave). We now show that these thresholds are also the solution to a standard clustering problem.

Proposition 4. Assume the supply function is linear. The optimal thresholds $z_{1}, \ldots, z_{N-1}$ for an $N$ - rating are the ones that minimize

$$
\sum_{k=1}^{N} \int_{z_{k-1}}^{z_{k}}\left(z-m\left(z_{k-1}, z_{k}\right)\right)^{2} d F(z)
$$

Proof. Taking derivatives with respect to $z_{k}$ gives first order condition (6).

The objective defined in the above proposition coincides with the popular $k$ - means criteria for clustering as introduced by MacQueen et al. (1967) which is commonly used in machine learning and statistics literature. Uniqueness of the thresholds is guaranteed when the distribution has log-concave density (Mease and Nair (2006).) This makes estimating the optimal thresholds a trivial task, as many software programs incorporate algorithms to solve this problem. Additionally, when combining this result with the one in Lemma 4, the simple algorithm can give a market designer a good place to start, given that the above thresholds will be the lower bound or upper bound, depending on the properties of the supply functions.

\subsection{Consumer and Producer Surplus}

The above thresholds maximize total surplus, however it might be the case that the certifier has a different objective, such as maximizing consumer surplus or profits. How would the corresponding thresholds compare the ones obtained above? The answer depends again on properties of the supply function. We have shown that consumer surplus moves together with total output. In the case of concave supply, total output decreases with information, so optimal thresholds are at the extremes of the distribution. When supply is linear, total quantity is independent of the amount of information (see Proposition 1), so consumer surplus is the same for any threshold. This implies that the optimal threshold is also the one that maximizes profits. More generally, total output will vary as the thresholds change. The following Lemma provides a characterization. 
Lemma 5. $d Q(\boldsymbol{z}) / d z_{k}$ has the same sign as

$$
\frac{z_{k}-M_{k}}{M_{k+1}-M_{k}} s^{\prime}\left(p_{k}\right)+\frac{M_{k+1}-z_{k}}{M_{k+1}-M_{k}} s^{\prime}\left(p_{k+1}\right)-\frac{\int_{p_{k}}^{p_{k+1}} s^{\prime}(p) d p}{p_{k+1}-p_{k}}
$$

Proof. See Appendix.

It is useful to compare this expression to equation (5) that corresponds to the optimal thresholds. Both represent expected values with the same lotteries, but with different functions. In parallel to Lemma 4 , it is immediate to prove:

Lemma 6. The certification thresholds $z_{1}, \ldots, z_{N-1}$ that maximize total output and consumer surplus satisfy $z_{k}=\left(M_{k}+M_{k+1}\right) / 2$ when $s^{\prime}(p)$ is linear, and is lower than (resp. higher than) this value when $s^{\prime}(p)$ is concave (resp. convex).

A comparison of equations (5) and (8) allows us provide some global comparison between the corresponding thresholds for the convex case, in parallel to Proposition 3.

Proposition 5. Let $\boldsymbol{z}^{o}=\left(\boldsymbol{z}_{\mathbf{1}}^{o}, \ldots, \boldsymbol{z}_{\mathbf{N - 1}}^{o}\right)$ be the thresholds that maximize total surplus and $\boldsymbol{z}^{c}=\left(z_{1}^{c}, \ldots, z_{N-1}^{c}\right)$ the ones that maximize consumer surplus. Suppose that the distribution of qualities $F(z)$ has log-concave density and the supply function is convex and $s^{\prime}(p)$ concave. Then $\boldsymbol{z}^{c}$ is pointwise smaller than $\boldsymbol{z}^{o}$.

To illustrate the above results, consider a simple example. Suppose the supply function $s(p)=p^{\theta}$ (cost function $\left.c(q)=q^{1+\theta} /(1+\theta)\right)$. For $0<\theta<1, s(\cdot)$ is concave, so consumers prefer no thresholds. For $\theta=1$, consumers are indifferent and the optimal thresholds also maximize profits. For $1<\theta \leq 2$, supply is convex but $s^{\prime}(\cdot)$ concave, so by the previous proposition consumers prefer lower thresholds. For $\theta>2, s^{\prime}(\cdot)$ is also convex, so this proposition does not apply. The following proposition and its corollary provide more general local results that also cover this case.

Proposition 6. Let $\boldsymbol{z}=\left(z_{1}, \ldots, z_{N-1}\right)$ be the thresholds that maximize total surplus.

1. If $s$ is convex and $s^{\prime \prime}(p) / s^{\prime}(p)$ is decreasing (resp. increasing) in $p$. Then $d Q(\boldsymbol{z}) / d z_{k}$ is negative (resp. positive) at $\boldsymbol{z}$.

2. If $s$ is concave and $s^{\prime \prime}(p) / s^{\prime}(p)$ is decreasing (resp. increasing) in $p$. Then $d Q(\boldsymbol{z}) / d z_{k}$ is positive (resp. negative) at $\boldsymbol{z}$.

Proof. See Appendix. 
The previous proposition can be used to sign the local behavior of consumer and producer surplus at any optimal threshold. Given that the derivative of total surplus is zero at any of the optimal thresholds $z_{k}$, derivatives of consumer surplus and producer surplus have the opposite signs. Moreover, derivative of consumer surplus has the same sign as $d Q / d z$. Hence the following corollary follows.

Corollary 2. Let $\boldsymbol{z}=\left(z_{1}, \ldots, z_{N-1}\right)$ be the thresholds that maximize total surplus.

1. If $s$ is convex and $s^{\prime \prime}(p) / s^{\prime}(p)$ is decreasing (resp. increasing) in $p$. Then the derivative of consumer surplus, $d C S(\boldsymbol{z}) / d z_{k}$, is negative (resp. positive) and the derivative of producer surplus, $d P S(\boldsymbol{z}) / d z_{k}$, is positive (resp. negative) at $\boldsymbol{z}$.

2. If $s$ is concave and $s^{\prime \prime}(p) / s^{\prime}(p)$ is decreasing (resp. increasing) in $p$. Then $d C S(\boldsymbol{z}) / d z_{k}$ is positive (resp. negative) and $d P S(\boldsymbol{z}) / d z_{k}$ is negative (resp. positive) at $\boldsymbol{z}$.

Going back to the previous example of homogeneous supply, $s^{\prime \prime}(p) / s^{\prime}(p)=(\theta-1) / p$ which is decreasing for convex supply functions $(\theta>1)$. Applying the first case in the corollary, this implies that starting at the surplus maximizing thresholds, consumers prefer lower thresholds while producers prefer higher ones. This highlights a tension in the choice of ratings systems between consumers and producers.

\section{Distribution Functions and Optimal Thresholds}

The thresholds defined by equation (6) are determined solely by the distribution of seller quality for the case of linear supply functions. In this section, we examine the impact of skewness on optimal thresholds. Our analysis is restricted to the case of linear supply function, though we conjecture it can be extended to more general supply. Our first result considers a weak notion of skewness (mean vs. median) but restricted to certification ratings $(N=2)$. Our second result relates to a stronger skewness order, the convex/concave order, applied to arbitrary $N$. Finally, we calculate optimal thresholds for a series of distribution functions and provide measures of surplus attainment as we increase the number of partitions. As an application, we solve for the optimal threshold for a distribution of seller quality from eBay.

To simplify notation in the case where $N=2$, we let $z^{*}$ denote the optimal threshold and $z_{L}$ and $z_{H}$ the conditional means below and above this threshold, respectively. The condition given in equation (6) implies

$$
z^{*}=\frac{1}{2}\left(z_{L}\left(z^{*}\right)+z_{H}\left(z^{*}\right)\right)
$$

which can be used to relate this threshold to properties of the distribution. Consider first the case of a symmetric distribution, i.e., where the median, $z_{\text {median }}$, equals the mean, $\bar{z}$. Since for 
any $z^{*}, F\left(z^{*}\right) z_{L}+\left(1-F\left(z^{*}\right)\right) z_{H}=\bar{z}$, setting the threshold $z^{*}=\bar{z}=z_{\text {median }}$ would satisfy the above condition. For skewed distributions $F(z)$, the following result can be proved:

Proposition 7. Suppose there is a unique point $z^{*}$ satisfying (9) and $\bar{z}$ is greater (resp. smaller) than $z_{\text {median. }}$. Then $z^{*}$ is greater (resp. smaller) than $\bar{z}$.

Proof. See Appendix.

The above proposition shows that if a distribution is skewed, the optimal threshold when $N=2$ will be even more skewed in the same direction. Table 1, shows the optimal threshold for a series of distributions, as well as the corresponding fraction of certified sellers. Except for the last case, all distributions are skewed to the right so according to Proposition 7 , $z^{*}>\bar{z}>z_{\text {median }}$ and it is optimal to have a small share of sellers certified. This is shown in the fifth column of our table. As an example, for the Pareto distributions only a small fraction should get certified, $5 \%$ when the power parameter is 3 and $9 \%$ when the power parameter is $4 .{ }^{17}$ For the exponential distribution, only $20 \%$ of sellers should be certified regardless of the hazard rate.

We now turn to the case of general $N$ under a stronger skewness order, the convex (concave) order, proposed originally by Van Zwet (1964).

Definition. Distribution $\tilde{F}$ is more skewed to the right than $F$ if $\tilde{F}^{-1}(F(x))$ is convex, equivalently, there exists an increasing convex function $g(x)$ such that $\tilde{F}(g(x))=F(x){ }^{18}$

We can think of this ordering as stretching to the right the quality scale with the transformation $g(x)$. As an example, if $F$ is a uniform distribution in $[0,1]$ and $g(x)=x^{2}$, then $\tilde{F}\left(x^{2}\right)=x$ or equivalently, $\tilde{F}(x)=x^{1 / 2}$.

Proposition 8. Suppose the supply function is linear. Let $F$ be a distribution with log-concave density and $\tilde{F}$ a distribution such that $\tilde{F}(g(z))=F(z)$, where $g$ is a strictly convex increasing function. Let $\left\{l_{k}\right\}$ be the optimal thresholds for $F$ and $\left\{g\left(z_{k}\right)\right\}$ the optimal thresholds for $\tilde{F}$. Then $z_{k}>l_{k}$ for all $k$.

Proof. See appendix.

This proposition implies that for all $k, \tilde{F}\left(g\left(z_{k}\right)\right)=F\left(z_{k}\right)>F\left(l_{k}\right)$ so the percentiles defined by the two optimal thresholds are ordered. In particular, for a 2-tier certification rating, the share of certified firms should be lower for distribution $\tilde{F}$. An example is given in Table 1 for the case of power distributions $F(z)=z^{\alpha}$. It is easily shown that the distribution

\footnotetext{
${ }^{17}$ When $\alpha \leq 2$, the value of $z^{*}$ is undefined, as total surplus is strictly increasing in $z^{*}$ in all the support.

${ }^{18}$ Note that this definition implies that $F^{-1}(F(x))=g^{-1}(x)$ is concave.
} 
with $\alpha=0.5$ is more skewed to the right than the one with $\alpha=2 .{ }^{19}$ Consistently with the previous proposition, the share of certified sellers is lower when $\alpha=0.5$.

We now examine how the surplus gap between the full-information and no-information settings changes as the number of groups in a partition increases for the case of linear supply. In this case, we have shown that total output $Q$ remains unchanged and so does consumer surplus. Therefore, the gap in total surplus equals the gap in total profits. For a firm with perceived quality $z$, profits are equal to $p(z)^{2} / 2=(P(Q)+z)^{2} / 2$. Therefore, for any distribution $G$ of expected quality, total profits are

$$
\begin{aligned}
\Pi & =\frac{1}{2} \int(P(Q)+z)^{2} d G(z) \\
& =\frac{1}{2} P(Q)^{2}+P(Q) \bar{z}+\frac{1}{2} \int z^{2} d G(z)
\end{aligned}
$$

The first two terms do not depend on $G$ and thus on the partition. For full information, the distribution of means $G=F$, so, $\Pi=\frac{1}{2} P(Q)^{2}+P(Q) \bar{z}+\frac{1}{2} \int z^{2} d F(z)$. Therefore, the surplus gap with respect to full information is $\Delta \Pi=\frac{1}{2}\left(\int z^{2} d F(z)-\int z^{2} d G(z)\right)$ for any distribution of expected quality $G$. In particular, the total gap with respect to the no-information case is $\Delta \Pi=\frac{1}{2}\left(\int z^{2} d F(z)-\bar{z}^{2}\right)$. For a threshold partition $\left(z_{1}, \ldots, z_{N-1}\right)$, where $G$ has $N$ mass points at the conditional means $M_{1}, \ldots, M_{N}$

$$
\begin{aligned}
\Delta \Pi & =\frac{1}{2} \sum_{k=1}^{N} \int_{z_{k-1}}^{z_{k}}\left(z^{2}-M_{k}^{2}\right) d F(z) \\
& =\frac{1}{2} \sum_{k=1}^{N} \int_{z_{k-1}}^{z_{k}}\left(z-M_{k}\right)^{2} d F(z) .
\end{aligned}
$$

This corresponds again to the loss function used in $k$-means clustering, given that at the optimal thresholds, as defined earlier, the expected values $\mathrm{M}_{k}$ are precisely the centroids of the corresponding intervals $\left[z_{k-1}, z_{k}\right]$. Table 1 reports the share of the total surplus gaps that is closed with partitions of different sizes $n$. As can be seen from the calculations a one-threshold (two-group) partition closes from near $50 \%$ to almost $80 \%$ of the surplus gap, depending on the underlying distribution of qualities. The gains are diminishing as the number of thresholds increases. Even though total surplus increases with the number of tiers, our numerical results suggest that most gains are attained with a small number of ratings. As a result, the market designer should weigh in the cost of having a more complicated information structure against the diminishing return of having more tiers. While we do not model the cost of providing more

\footnotetext{
${ }^{19}$ Take $g(x)=x^{4}$.
} 
Table 1: Optimal Thresholds

\begin{tabular}{|c|c|c|c|c|c|c|c|c|}
\hline \multirow{2}{*}{ Distribution } & \multirow{2}{*}{ Case } & \multirow{2}{*}{ Mean/Median } & \multirow{2}{*}{$z^{*}$} & \multirow{2}{*}{$1-F\left(z^{*}\right)$} & \multicolumn{4}{|c|}{ Share of Surplus Gap Closed } \\
\hline & & & & & $n=2$ & $n=3$ & $n=5$ & $n=10$ \\
\hline \multirow[t]{2}{*}{ Pareto } & $\alpha=3$ & 1.19 & 2.73 & 0.05 & 0.46 & 0.68 & 0.84 & 0.94 \\
\hline & $\alpha=4$ & 1.12 & 1.84 & 0.09 & 0.54 & 0.74 & 0.89 & 0.97 \\
\hline Exponential & all & 1.45 & & 0.20 & 0.65 & 0.82 & 0.93 & 0.98 \\
\hline \multirow{2}{*}{$\begin{array}{c}F(z)=z^{\alpha} \\
z \in[0,1]\end{array}$} & $\alpha=0.5$ & 1.32 & 0.41 & 0.36 & 0.77 & 0.90 & 0.97 & 0.99 \\
\hline & $\alpha=2$ & 0.94 & 0.62 & 0.62 & 0.72 & 0.87 & 0.95 & 0.99 \\
\hline \multirow{2}{*}{$\begin{array}{l}\text { Log-normal } \\
\quad(\mu=0)\end{array}$} & $\sigma=0.25$ & 1.03 & 1.09 & 0.36 & 0.63 & 0.81 & 0.92 & 0.98 \\
\hline & $\sigma=1$ & 1.64 & 4.25 & 0.07 & 0.55 & 0.75 & 0.89 & 0.97 \\
\hline eBay (Kernel) & band $=0.2$ & 0.97 & 0.63 & 0.63 & 0.66 & 0.83 & 0.93 & 0.98 \\
\hline
\end{tabular}

Note: The above calculations correspond to the linear supply case.

detailed information for the market designer or consumer's cost of analyzing and understanding detailed information, our results suggest that small costs could justify simple optimal rating systems that include only limited number of tiers, as usually seen in practice.

Following McAfee (2002); Hoppe et al. (2011); Shao (2016), it is possible to find lower bounds for the share of the surplus gap that is closed by a one-threshold partition. While these papers consider the case of one-to-one matching, our setup with linear supply can be conveniently mapped into this setting. Let $z$ represent the type of a seller, $\theta$ that of a buyer, and $m(z, \theta)=z \theta$ the surplus of this match. Furthermore, assume that both seller and consumer types have the same distribution $F$. This is a special case of the class of matching problems considered in the above papers. With complete information, the optimal matching is assortative, with total value $W=\int z^{2} d F(z)$. In contrast, in a two-group partition with threshold $z^{*}$ and conditional means $z_{L}$ and $z_{H}$, there is random matching within each partition, resulting in total value $\tilde{W}=F\left(z^{*}\right) z_{L}^{2}+\left(1-F\left(z^{*}\right)\right) z_{H}^{2}$. At the other extreme, with no information and pure random matching, the value is simply $\bar{z}^{2}$. The gap between these values is proportional to the corresponding ones in our setting, as given by equation (10), so the relative gain from a one-threshold partition is identical in both cases. ${ }^{20}$

McAfee (2002) finds that for the class of distribution functions with log concave densities, the relative gain is at least $1 / 2$. For this class of distributions, Hoppe et al. (2011) show that $\tilde{W} \geq \frac{3}{4} W$. They also find that the same bound as McAfee (2002) applies to distributions with decreasing hazard rate.

These results show that a very simple rating system consisting of a single certification threshold can achieve a considerable share of the gains from full information. This suggests that the added cost or complexity of a more elaborate design might not be compensated by

\footnotetext{
${ }^{20}$ More generally, the relative gain from any information structure is the same.
} 
the gains. This could explain the popularity of such simple schemes among market designers.

\section{Optimal Threshold for eBay}

Nosko and Tadelis (2015) provides a quality measure given by the percentage time a seller got positive feedback (as opposed to negative or none). The distribution of this statistic across sellers is given in Figure 2 together with a density kernel estimator. ${ }^{21}$ If we interpret this statistic as an ex-ante probability of a good (vs. a bad) experience and the expected utility from this purchase as $(1-P($ good $)) u(b a d)+P($ good $) u($ good $)$, then expected utility is an affine transformation of the probability of a good experience. Based on this interpretation, we can use this distribution to calculate the optimal certification threshold, as done for other distributions above. Table 1 reports the results for the kernel estimate of this distribution. According to our calculations, more than $65 \%$ of sellers should be certified, closing about $63 \%$ of the surplus gap. The exercise highlights the importance of skewness in the distribution of qualities. While our mapping of this data to a quality distribution is admittedly quite special, it suggests that filtering out the lower end of the distribution so to avoid bad experiences could provide considerable value. ${ }^{22}$

\section{Extensions}

In this section we consider a series of extensions. First, we consider heterogeneous preference for quality. Second, we show results for Cournot competition. Third, we analyze the effect of entry.

\subsection{Heterogeneous Preference for Quality}

We examine briefly the determination of optimal thresholds when consumers differ in their preferences for quality for the case where $N=2$. Suppose consumers' preferences are given by the utility function $u=\theta z+\theta_{0}-p$ for a good of quality $z$, a la Mussa and Rosen (1978). Consumers differ in their preference for quality $\theta$ and for the value they assign the inside vs. outside good $\theta_{0}$, which is distributed in the population according to some joint distribution $\Psi\left(\theta, \theta_{0}\right)$. As earlier, firm qualities $z$ are distributed according to cdf $F(z)$. For simplicity, we restrict our analysis to a partition of sellers into two groups defined by threshold $z^{*}$ with

\footnotetext{
${ }^{21}$ The data for the histogram come directly from Table 4 in Nosko and Tadelis (2015).

${ }^{22}$ Also note that here we make very special assumptions in terms of supply and demand functions; for example, we assume a linear the supply function, while if the supply function is convex, the optimal $z^{*}$ estimated is just a lower bound for the actual $z^{*}$.
} 


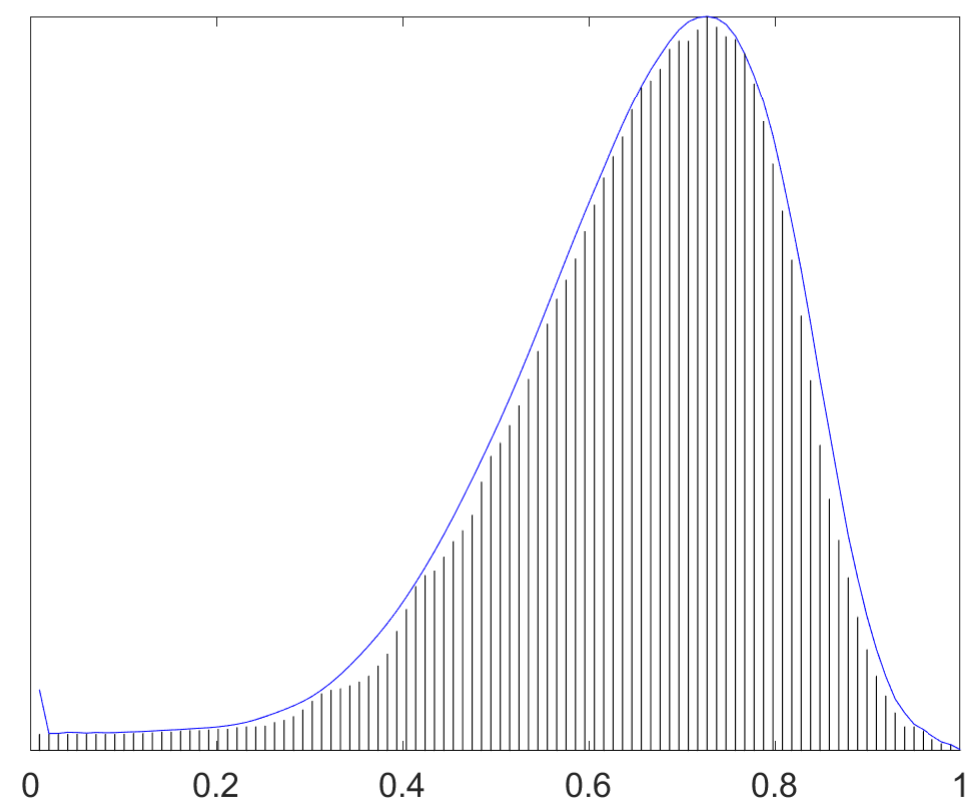

Figure 2: Distribution of \% Positive Responses for eBay sellers

qualities $z_{L}$ and $z_{H}$, respectively. Given prices $p_{L}$ and $p_{H}$, consumers will be split into three groups: those that do not consume and those that consume either the $H$ or $L$ product, with demands $D_{H}\left(p_{L}, p_{H}\right)$ and $D_{L}\left(p_{L}, p_{H}\right)$, respectively. These will be equilibrium prices provided that $D_{H}\left(p_{L}, p_{H}\right)=\left(1-F\left(z^{*}\right)\right) q\left(p_{H}\right)$ and $D_{L}\left(p_{L}, p_{H}\right)=F\left(z^{*}\right) q\left(p_{L}\right)$. As in our previous case, there is a unique equilibrium under fairly general conditions.

Lemma 7. The optimal choice of threshold $z^{*}$ satisfies the following first-order necessary condition:

$$
\Pi\left(p_{H}\right)-\Pi\left(p_{L}\right)=\left(z^{*}-z_{L}\right) \theta_{L} q_{L}+\left(z_{H}-z^{*}\right) \theta_{H} q_{H},
$$

where $\theta_{L}$ is the average preference for quality of consumers who purchase the $L$ product, and $\theta_{H}$ of those who purchase the $H$ product.

Proof. See Appendix.

This formula has an intuitive explanation. The first term is the loss of profits by those firms that transition from the $H$ to the $L$ group, when $z^{*}$ is marginally increasing. The second term measures the effect of the increase in the averages $z_{L}$ and $z_{H}$ as $z^{*}$ is increased, valued at the quality preference of the average consumer in each group and weighted by their respective market sizes. 


\section{Vertical Differentiation with Inelastic Supply}

To establish further results, we consider the canonical model of vertical differentiation where consumers differ only in their preference for quality $\theta$ and where firms supply inelastically one unit of output. ${ }^{23}$ Given equilibrium prices $p_{L}$ and $p_{H}$, all consumers above a threshold $\theta^{*}$ buy an $H$ product, while all those between $\underline{\theta}$ and $\theta^{*}$ buy an $L$ product, where $\underline{\theta} z_{L}=p_{L}$ and $\theta^{*}\left(z_{H}-z_{L}\right)=p_{H}-p_{L}$. Substituting in equation (11) gives the condition

$$
\left(z^{*}-z_{L}\right)\left(\theta^{*}-\theta_{L}\right)=\left(z_{H}-z^{*}\right)\left(\theta_{H}-\theta^{*}\right) .
$$

Notice that this is a modified version of equation (6), where the gaps between $z^{*}$ and the respective means are weighted by the corresponding preference gaps. This highlights the role of the complementarities between average quality and preference for quality in the determination of the optimal threshold. In particular, when both distributions are symmetric, this also implies that the optimal threshold $z^{*}$ (and also $\theta^{*}$ ) will equal the corresponding mean. Moreover, when $z$ and $\theta$ have the same distribution, the optimal threshold is also given by our baseline condition, as given in equation (6). ${ }^{24}$ As an example, if both have uniform distributions, then when $\theta^{*}=z^{*}=1 / 2$, this condition will hold.

\subsection{Cournot Competition}

Here we restrict the analysis to a fixed set of firms, without considering explicitly the effect of changes in $z^{*}$ on entry. There is a total of $N$ firms (per consumer), and given signal $z^{*}$, a fraction $F\left(z^{*}\right)$ in the first group and $\left(1-F\left(z^{*}\right)\right)$ in the second. The demand structure is the same as in the competitive case considered above. Assume firms face a constant marginal cost $c$ regardless of their type. The equilibrium conditions are

$$
\begin{aligned}
M R_{H} & =P^{\prime}(Q) q_{h}+P(Q)+z_{H}=c \\
M R_{L} & =P^{\prime}(Q) q_{l}+P(Q)+z_{L}=c .
\end{aligned}
$$

Multiplying each equation by the number of firms in the respective group and adding up, we get

$$
P^{\prime}(Q) Q+N P(Q)+N \bar{z}=N c
$$

\footnotetext{
${ }^{23}$ This can be reinterpreted as a one-to-one matching environment with surplus function $\theta z$.

${ }^{24}$ It is interesting to note that when all consumers have the same preference for quality and supply is inelastic, welfare is independent of $z^{*}$, as the average product quality is not affected by its choice.
} 
where $\bar{z}$ is the mean quality for the $N$ firms. Interestingly, this equation determines $Q$ independently of the signal threshold $z^{*}$, as in the case of perfect competition.

Another implication of the invariance of total output is that consumer surplus does not change, as in the case of linear supply with $z^{*}$. This occurs because price increases capture exactly the change in average quality in each group. It follows that optimal thresholds solve the maximization problem (3), so they are identical to those obtained above for the linear case. $^{25}$

\subsection{Entry}

In our previous analysis we did not consider explicitly the effect of changes in $z^{*}$ on entry. Many of our results extend to settings where the distribution of qualities of firms is not affected by entry. We discuss here two scenarios: one where entrants are ex-ante differentiated and one when they are ex-ante homogeneous.

Consider first the case of differentiated entrants. Our analysis extends without modification in the following scenario. Suppose there is a mass $N$ of entrants that are differentiated in qualities $z$ and fixed (or entry) costs $f$. Assume qualities are independent from fixed costs and are given by distribution $F$ and $\Phi$, respectively. For a given threshold partition $z^{*}$, we can define the aggregate supply functions $S_{L}$ and $S_{H}$ as follows. Let $S_{H}(p)=s(p) N_{H}(p)$, where $N_{H}(p)=N\left(1-F\left(z^{*}\right)\right) \Phi(\pi(p))$. This supply function combines the effect of prices on the intensive and extensive margin. We can define similarly $S_{L}(p)$. Our analysis remains unchanged if we substitute $s(p)$ by $\hat{s}(p)=s(p) N \Phi(f(p))$, so total supplies are $S_{L}(p)=$ $F\left(z^{*}\right) \hat{s}(p)$ and $S_{H}(p)=\hat{s}(p)\left(1-F\left(z^{*}\right)\right) \cdot{ }^{26}$

For the homogeneous case, assume there is a set $N$ of potential entrants that draw their qualities independently from distribution $F$ upon entry, after paying an entry cost $f$, which is distributed according to cdf $\Phi(f)$. For fixed output, improved information results in a mean-preserving spread of expected qualities and thus prices. Given that profit functions are convex in prices, this results in an increase in expected profits and a consequent increase in entry. In the case of linear supply, where in the absence of entry, total output does not change, additional entry results in an increase in total output and thus consumer surplus. In the case of concave supply, we have seen that total output decreases. This increases profits over and beyond what is produced by the mean-preserving spread of average qualities, thus

\footnotetext{
${ }^{25}$ We have considered here quantity competition. For a model of price competition with partially informed consumers see Moscarini and Ottaviani (2001).

${ }^{26}$ The properties of these modified supply functions will now depend both on the individual supply functions and the distribution of fixed costs. There exist assumptions on the latter that will guarantee that the modified supply functions are linear, convex, or concave when these properties hold for the original supply functions.
} 
inducing entry, mitigating, if not totally undoing, the drop in total output that would result in the absence of entry. Finally, note that if all potential entrants were to have the same entry cost, all surplus gains from improved information would accrue to consumers, as expected and average profits would remain unchanged. The above results apply in particular to the effect of introducing a certification mechanism in a market where there is none.

\section{$7 \quad$ Final Remarks}

This paper considered the optimal design of quality ratings in markets with adverse selection. Ratings reallocate demand across producers, impacting the average quality of goods consumed but also average cost. The optimal thresholds in a discrete rating system optimize this tradeoff. Optimal ratings thus depend on characteristics of the market, given by the distribution of producers quality, the elasticity of supply and consumer preferences. We find that the optimal thresholds in the case of a convex (resp. concave) supply function are pointwise higher (resp. lower) than those in the linear case. Intuitively, in case of a simple certification rating with two groups, more elastic supply leads to a higher threshold and lower share of certified sellers. We also find that skewness in the distribution of firm qualities matters for optimal ratings, which move in the direction of the skew.

We have given a simple characterization for the optimal thresholds in the case of linear supply, or Cournot competition with constant marginal cost, as the solution to a standard $k$-means clustering problem. Our results thus provide a straightforward and easy-to-compute method for the design of rating systems. This method is used to derive bounds on the performance of the rating system as a function of the number of categories. As an example, we find that for the exponential family of distributions, $65 \%$ of the surplus gains from full information can be achieved with only two categories. In addition we provide general bounds for the case of distribution functions with log-concave density. The large gains in surplus with a very simple threshold mechanism suggest that the added cost of a more complex one might not be compensated by the gains. This could explain the popularity of these simple schemes among market designers.

As for the distribution of surplus, we find that there is a tension between the interest of consumers and producers in the design of a rating mechanism. When supply is concave, better information hurts consumers and benefits producers. More generally, our results suggest the thresholds that maximize producer surplus and those that maximize consumer surplus are on opposite sides of those maximizing total surplus.

Our preliminary analysis of the vertical differentiation model suggests that preferences for quality can be an important factor in determining optimal thresholds. One might conjecture 
that, in parallel to our results on the supply side, more convexity (resp. concavity) in the distribution of consumer types increases (resp. decreases) the gains from more assortative matching, thus leading to higher thresholds. This question and further extensions on the demand side, such as including scope for horizontal differentiation, are subject for future research. Other extensions worth considering are a more detailed modeling of entry, following results obtained in the empirical literature Hui et al. (2018). Finally, we have abstracted from moral hazard considerations which can be important in some settings. Exploring the impact these may have on the design of optimal ratings is subject for future research. 


\section{References}

BAJARI, P. AND A. Hortacsu (2004): "Economic insights from internet auctions," Journal of Economic Literature, 42, 457-486. 8

Bergemann, D. And A. Bonatti (2019): "Markets for information: An introduction," Annual Review of Economics, 11. 6

Bergemann, D., B. Brooks, And S. Morris (2015): "The Limits of Price Discrimination," American Economic Review, 105, 921-57. 6

Bergemann, D. And S. Morris (2013): "Robust predictions in games with incomplete information," Econometrica, 81, 1251-1308. 7

_ (2016): "Bayes correlated equilibrium and the comparison of information structures in games," Theoretical Economics, 11, 487-522. 7

_ (2017): "Information design: A unified perspective," . 7

Bergemann, D. And M. Pesendorfer (2007): "Information structures in optimal auctions," Journal of economic theory, 137, 580-609. 6

Bhaskar, U., Y. Cheng, Y. K. Ko, and C. Swamy (2016): "Hardness results for signaling in bayesian zero-sum and network routing games," in Proceedings of the 2016 ACM Conference on Economics and Computation, ACM, 479-496. 7

Bhaskar, V., C. D. Thomas, ET AL. (2017): The design of credit information systems, Centre for Economic Policy Research. 7

BoARD, S. (2009): "Revealing information in auctions: the allocation effect," Economic Theory, $38,125-135.6$

Cabral, L. And A. Hortacsu (2010): "The dynamics of seller reputation: Evidence from ebay*," The Journal of Industrial Economics, 58, 54-78. 8

Che, Y.-K. And J. Hörner (2018): "Recommender Systems as Mechanisms for Social Learning," The Quarterly Journal of Economics, 133, 871-925. 7

Chevalier, J. A. And D. Mayzlin (2006): "The effect of word of mouth on sales: Online book reviews," Journal of marketing research, 43, 345-354. 8 
De Marzo, P., I. Kremer, and A. Skrzypacz (2018): "Testing Design and Minimum Standards," American Economic Review, forthcoming. 7

Dellarocas, C., F. Dini, and G. Spagnolo (2006): "Designing reputation (feedback) mechanisms," . 8

Dranove, D. And G. Z. Jin (2010): "Quality disclosure and certification: Theory and practice," Journal of Economic Literature, 48, 935-963. 6

Dworczak, P. And G. Martini (2019): "The Simple Economics of Optimal Persuasion," Journal of Political Economy, forthcoming. 7, 14

EkmeKci, M. (2011): "Sustainable reputations with rating systems," Journal of Economic Theory, 146, 479-503. 7

Elfenbein, D. W., R. Fisman, And B. McManus (2015): "Market structure, reputation, and the value of quality certification," American Economic Journal: Microeconomics, 7, 83-108. 8

Fan, Y., J. Ju, and M. XiaO (2013): "Losing to Win: Reputation Management of Online Sellers,". 8

Filippas, A., J. J. Horton, And J. Golden (2018): "Reputation inflation," in Proceedings of the 2018 ACM Conference on Economics and Computation, ACM, 483-484. 8

Ganuza, J.-J. And J. S. Penalva (2010): "Signal Orderings Based on Dispersion and the Supply of Private Information in Auctions," Econometrica, 78, 1007-1030. 3, 9, 11

Gentzkow, M. and E. Kamenica (2016): "A Rothschild-Stiglitz Approach to Bayesian Persuasion," American Economic Review, 106. 3, 9

Harbaugh, R. And E. Rasmusen (2018): "Coarse grades: Informing the public by withholding information," American Economic Journal: Microeconomics, 10, 210-35. 7

Hoppe, H. C., B. Moldovanu, and E. Ozdenoren (2011): "Coarse matching with incomplete information," Economic Theory, 47, 75-104. 6, 7, 12, 22

Hörner, J. AND N. S. LAmbert (2016): "Motivational ratings," . 7

Hui, X., M. Saeedi, Z. Shen, And N. Sundaresan (2016): "Reputation and regulations: evidence from eBay," Management Science, 62, 3604-3616. 8 
Hui, X., M. Saeedi, G. Spagnolo, and S. Tadelis (2018): "Certification, Reputation and Entry: An Empirical Analysis," Working Paper 24916, National Bureau of Economic Research. 2, 28

Hui, X., M. Saeedi, And N. Sundaresan (2017): "Adverse Selection or Moral Hazard: An Empirical Study," Working paper. 3

Jin, G. Z. AND P. LeSLIE (2003): "The effect of information on product quality: Evidence from restaurant hygiene grade cards," The Quarterly Journal of Economics, 118, 409-451. 8

KAmenicA, E. (2018): "Bayesian persuasion and information design," Annual Review of Economics. 7

Kovbasyuk, S. And G. Spagnolo (2017): "Memory and markets," . 7

Kremer, I., Y. Mansour, And M. Perry (2014): "Implementing the "wisdom of the crowd"," Journal of Political Economy, 122, 988-1012. 7

LiU, Q. AND A. Skrzypacz (2014): "Limited records and reputation bubbles," Journal of Economic Theory, 151, 2-29. 7

Lizzeri, A. (1999): "Information revelation and certification intermediaries," The RAND Journal of Economics, 214-231. 6

MacQueen, J. ET AL. (1967): "Some methods for classification and analysis of multivariate observations," in Proceedings of the fifth Berkeley symposium on mathematical statistics and probability, Oakland, CA, USA, vol. 1, 281-297. 5, 17

McAfee, R. P. (2002): "Coarse matching," Econometrica, 70, 2025-2034. 7, 22

Mease, D. And V. N. NAIR (2006): "Unique optimal partitions of distributions and connections to hazard rates and stochastic ordering," Statistica Sinica, 1299-1312. 17, 33

Moscarini, G. And M. Ottaviani (2001): "Price competition for an informed buyer," Journal of Economic Theory, 101, 457-493. 26

Mussa, M. And S. Rosen (1978): "Monopoly and product quality," Journal of Economic theory, 18, 301-317. 23 
Nosko, C. And S. Tadelis (2015): "The limits of reputation in platform markets: An empirical analysis and field experiment," Tech. rep., National Bureau of Economic Research. 8,23

Ostrovsky, M. And M. Schwarz (2010): "Information disclosure and unraveling in matching markets," American Economic Journal: Microeconomics, 2, 34-63. 7

Rothschild, M. And J. E. Stiglitz (1970): "Increasing risk: I. A definition," Journal of Economic theory, 2, 225-243. 13

Saeedi, M. (2014): "Reputation and Adverse Selection, Theory and Evidence from eBay," Working Paper. 8

Saeedi, M. and A. Shourideh (2019): "Optimal Rating Design," . 3

SchleE, E. E. (1996): "The Value of Information about Product Quality," The RAND Journal of Economics, 27, 803-815. 6, 12

Shao, R. (2016): "Generalized coarse matching," Games and Economic Behavior, 100, 142148. 22

TADELIS, S. (2016): "Reputation and feedback systems in online platform markets," Annual Review of Economics, 8, 321-340. 8

Van Zwet, W. (1964): "Convex transformations of random variables, Math," Centrum, Amsterdam, 1964. 20

VelLodi, N. (2019): "Ratings Design and Barriers to Entry," . 7

WiLson, R. (1989): "Efficient and competitive rationing," Econometrica: Journal of the Econometric Society, 1-40. 7

Wu, C., H. Che, T. Y. Chan, And X. Lu (2015): "The economic value of online reviews," Marketing Science, 34, 739-754. 8 


\section{Appendix. Proofs}

\section{Proof of Lemma 3}

First note that

$$
\begin{aligned}
\left(P(Q)+z_{k}\right)\left(q_{k+1}-q_{k}\right) & =\left(P(Q)+M_{k+1}-M_{k+1}+z_{k}\right) q_{k+1} \\
& -\left(P(Q)+M_{k}-M_{k}+z_{k}\right) q_{k} \\
& =p_{k+1} q_{k+1}-p_{k} q_{k}-\left(M_{k+1}-z_{k}\right) q_{k+1}-\left(z_{k}-M_{k}\right) q_{k} .
\end{aligned}
$$

Substituting in (4) and rearranging gives:

$$
\left(M_{k+1}-z_{k}\right) q_{k+1}+\left(z_{k}-M_{k}\right) q_{k}=\pi_{k+1}-\pi_{k} .
$$

Equation (5) follows by substituting $\pi_{k+1}-\pi_{k}=\int_{p_{k}}^{p_{k+1}} s(p) d p$, using $q_{k+1}=s\left(p_{k+1}\right)$ and $q_{k}=s\left(p_{k}\right)$ and dividing through the left hand side by $\left(M_{k+1}-M_{k}\right)$ and the right hand side by the equivalent value $p_{k+1}-p_{k}$.

\section{Proof of Proposition 3 and Proposition 8}

We use the following properties of distributions with log-concave densities (see Lemma 1 in Mease and Nair (2006)):

$$
\begin{aligned}
& \mathbb{E}(z \mid s \leq z \leq s+d)-s \text { is decreasing in } s \text { for } d>0 \\
& s-\mathbb{E}(z \mid s-d \leq z \leq s) \text { is increasing in } s \text { for } d>0
\end{aligned}
$$

and these properties are preserved when conditioning on intervals.

Lemma 8. Suppose $F$ is a distribution with log-concave density and let $m(a, b)=E_{F}(z \mid a \leq z \leq b)$. Suppose the vector of thresholds $\left\{l_{k}\right\}_{k=1}^{N-1}$ satisfy

$$
l_{k}-m\left(l_{k-1}, l_{k}\right)=m\left(l_{k}, l_{k+1}\right)-l_{k}
$$

and let $z_{1}, \ldots, z_{N-1}$ be a vector such that

$$
z_{k}-m\left(z_{k-1}, z_{k}\right)>m\left(z_{k}, z_{k+1}\right)-z_{k}
$$


Then $z_{k}>l_{k}$ for all $k$.

To prove Lemma 8 we use first the following:

Claim. Under the assumptions of Lemma 8, suppose that for some $k, z_{k}<l_{k}$ and $z_{k+1}-z_{k} \geq$ $l_{k+1}-l_{k}$. Then $z_{k-1}<l_{k-1}$ and $z_{k}-z_{k-1} \geq l_{k}-l_{k-1}$.

Proof. Note that

$$
\begin{aligned}
z_{k}-m\left(z_{k-1}, z_{k}\right) & >m\left(z_{k}, z_{k+1}\right)-z_{k} \\
& \geq m\left(z_{k}, z_{k}+l_{k+1}-l_{k}\right)-z_{k} \\
& \geq m\left(l_{k}, l_{k+1}\right)-l_{k} \\
& =l_{k}-m\left(l_{k-1}, l_{k}\right) .
\end{aligned}
$$

The first inequality follows from (17), the second one from monotonicity of $m$, the third from (14), and the last by (16). Now consider $k-1$. We will show that $z_{k}-z_{k-1} \geq l_{k}-l_{k-1}$.

Suppose, by way of contradiction, that $z_{k}-z_{k-1}<l_{k}-l_{k-1}$. Then

$$
\begin{aligned}
z_{k}-m\left(z_{k-1}, z_{k}\right) & \leq l_{k}-m\left(l_{k}-\left(z_{k}-z_{k-1}\right), l_{k}\right) \\
& \leq l_{k}-m\left(l_{k-1}, l_{k}\right)
\end{aligned}
$$

where the first inequality follows from condition (15) and the second one from the monotonicity of $m$. This inequality contradicts (18), proving that $z_{k}-z_{k-1} \geq l_{k}-l_{k-1}$. Given that $z_{k}<l_{k}$, this also guarantees that $z_{k-1}<l_{k-1}$.

We now prove Lemma 8. Let $h$ denote the highest $k$ for which $z_{k}<l_{k}$. By the definition of $h, z_{h+1}-z_{h}>l_{h+1}-l_{h}$. Using inductively the previous claim, it follows that the same is true for all $k=1, \ldots, h$. For $k=1$, the claim would imply that $z_{0}<l_{0}$ which cannot be true if the distribution had a lower bound since in that case both $z_{0}$ and $l_{0}$ should equal this lower bound. For unbounded support, an argument similar to the one used in the claim can be used to generate a contradiction. This completes the proof.

\section{Proof of Proposition 3.}

Let $\left\{l_{k}\right\}$ denote the optimal thresholds for the linear supply function and $\left\{z_{k}\right\}$ those for the convex supply function. Lemma 4 (16) and (??) hold, so Lemma 8 proves the proposition. 


\section{Proof or Proposition 8.}

We first prove the following:

Lemma 9. Let $g\left(z_{1}\right), \ldots, g\left(z_{N-1}\right)$ be the optimal thresholds for $\tilde{F}$. Let $M_{k}=m\left(z_{k-1}, z_{k}\right)=$ $E_{F}\left(z_{k-1} \leq z \leq z_{k}\right)$. Then $z_{k}-M_{k}>M_{k+1}-z_{k}$.

Proof. Let $\tilde{M}_{k}=E_{\tilde{F}}\left(g\left(\tilde{z}_{k-1}\right) \leq z \leq g\left(\tilde{z}_{k}\right)\right)$. Note that by strict convexity of $g, \tilde{M}_{k}>g\left(M_{k}\right)$. It follows that

$$
\begin{aligned}
z-M_{k} & >z_{k}-g^{-1}\left(\tilde{M}_{k}\right) \\
& =g^{-1}\left(g\left(z_{k}\right)\right)-g^{-1}\left(\tilde{M}_{k}\right) \\
& =g^{-1}\left(\tilde{M}_{k+1}\right)-g^{-1}\left(g\left(z_{k}\right)\right) \\
& >M_{k+1}-z_{k}
\end{aligned}
$$

To prove the proposition, let the vector $\left\{l_{k}\right\}$ be the optimal thresholds for $F$ and $\left\{z_{k}\right\}$ the optimal thresholds for $\tilde{F}$. Equation (16) follows from the necessary condition for optimal thresholds and (??) follows by the previous lemma.

\section{Proof of Lemma 5}

Total output

$$
Q=\sum_{k=1}^{N}\left(F\left(z_{k}\right)-F\left(z_{k-1}\right)\right) s\left(p_{k}\right) .
$$

where $p_{k}=P(Q)+M_{k}$. Differentiating with respect to $z_{k}$ and using

$$
\begin{aligned}
\left(F\left(z_{k}\right)-F\left(z_{k-1}\right)\right) \frac{\partial M_{k}}{\partial z_{k}} & =f\left(z_{k}\right)\left(z_{k}-M_{k}\right) \\
\left(F\left(z_{k+1}\right)-F\left(z_{k}\right)\right) \frac{\partial M_{k+1}}{\partial z_{k}} & =f\left(z_{k}\right)\left(M_{k+1}-z_{k}\right)
\end{aligned}
$$




$$
\begin{aligned}
\frac{\partial Q}{\partial z_{k}} & =f\left(z_{k}\right)\left(s\left(p_{k}\right)-s\left(p_{k+1}\right)\right) \\
& +f\left(z_{k}\right)\left[s^{\prime}\left(p_{k}\right)\left(M_{k}-z_{k}\right)+s^{\prime}\left(p_{k+1}\right)\left(M_{k+1}-z_{k}\right)\right] \\
& +\sum_{k=1}^{N}\left(F\left(z_{k}\right)-F\left(z_{k-1}\right)\right) s^{\prime}\left(p_{k}\right) P^{\prime}(Q) \frac{\partial Q}{\partial z_{k}}
\end{aligned}
$$

so

$$
\frac{\partial Q}{\partial z_{k}}=\frac{f\left(z_{k}\right)\left[s\left(p_{k}\right)-s\left(p_{k+1}\right)+s^{\prime}\left(p_{k}\right)\left(M_{k}-z_{k}\right)+s^{\prime}\left(p_{k+1}\right)\left(M_{k+1}-z_{k}\right)\right]}{1-\sum_{k=1}^{N}\left(F\left(z_{k}\right)-F\left(z_{k-1}\right)\right) s^{\prime}\left(p_{k}\right) P^{\prime}(Q)}
$$

The denominator is positive since $s^{\prime}\left(p_{k}\right)>0$ and $P^{\prime}(Q)<0$ so $\partial Q / \partial z_{k}$ has the same sign as

$$
s\left(p_{k}\right)-s\left(p_{k+1}\right)+s^{\prime}\left(p_{k}\right)\left(M_{k}-z_{k}\right)+s^{\prime}\left(p_{k+1}\right)\left(M_{k+1}-z_{k}\right)
$$

and since $s\left(p_{k}\right)-s\left(p_{k+1}\right)=-\int_{p_{k}}^{p_{k+1}} s^{\prime}(p) d p$ and $p_{k+1}-p_{k}=M_{k+1}-M_{k}$, equation (8) follows.

\section{Proof of Proposition 6}

Letting $\alpha\left(z_{k}\right)=\frac{M_{k+1}-z_{k}}{M_{k+1}-M_{k}}$ we can rewrite Equation (5) as

$$
s\left(p_{k}\right)+\alpha\left(z_{k}\right)\left(s\left(p_{k+1}\right)-s\left(p_{k}\right)\right)=s\left(p_{k}\right)+\frac{\int_{p_{k}}^{p_{k+1}} s(p)-s\left(p_{k}\right) d p}{p_{k+1}-p_{k}}
$$

SO

$$
\alpha\left(z_{k}\right)=\frac{\int_{p_{k}}^{p_{k+1}} \frac{s(p)-s\left(p_{k}\right)}{s\left(p_{k+1}\right)-s\left(p_{k}\right)} d p}{p_{k+1}-p_{k}} .
$$

To evaluate $d Q / d z_{k}$ at the optimal thresholds $z_{1}, \ldots, z_{k}$ we rewrite, equation (8) in similar fashion using the expression for $\alpha\left(z_{k}\right)$ given by equation (19).

$$
\begin{aligned}
\frac{d Q}{d z_{k}} \stackrel{s}{=} \alpha\left(z_{k}\right)-\frac{\int_{p_{k}}^{p_{k+1}} \frac{s^{\prime}(p)-s^{\prime}\left(p_{k}\right)}{s^{\prime}\left(p_{k+1}\right)-s^{\prime}\left(p_{k}\right)} d p}{p_{k+1}-p_{k}} \\
=\frac{\int_{p_{k}}^{p_{k+1}} \frac{s(p)-s\left(p_{k}\right)}{s\left(p_{k+1}\right)-s\left(p_{k}\right)} d p-\int_{p_{k}}^{p_{k+1}} \frac{s^{\prime}(p)-s^{\prime}\left(p_{k}\right)}{s^{\prime}\left(p_{k+1}\right)-s^{\prime}\left(p_{k}\right)} d p}{p_{k+1}-p_{k}}
\end{aligned}
$$

so $d Q / d z_{k}$ has the same sign as

$$
\int_{p_{k}}^{p_{k+1}} \frac{s(p)-s\left(p_{k}\right)}{s\left(p_{k+1}\right)-s\left(p_{k}\right)} d p-\int_{p_{k}}^{p_{k+1}} \frac{s^{\prime}(p)-s^{\prime}\left(p_{k}\right)}{s^{\prime}\left(p_{k+1}\right)-s^{\prime}\left(p_{k}\right)} d p
$$


so a sufficient condition for $d Q / d z_{k}$ to be positive (negative) is that

$$
\frac{s(p)-s\left(p_{k}\right)}{s\left(p_{k+1}\right)-s\left(p_{k}\right)}-\frac{s^{\prime}(p)-s^{\prime}\left(p_{k}\right)}{s^{\prime}\left(p_{k+1}\right)-s^{\prime}\left(p_{k}\right)}>0(<0)
$$

For convex $s, s^{\prime}\left(p_{k+1}\right)-s^{\prime}\left(p_{k}\right)>0$ so we can rewrite this condition as

$$
\frac{s^{\prime}\left(p_{k+1}\right)-s^{\prime}\left(p_{k}\right)}{s\left(p_{k+1}\right)-s\left(p_{k}\right)}-\frac{s^{\prime}(p)-s^{\prime}\left(p_{k}\right)}{s(p)-s\left(p_{k}\right)}>0(<0)
$$

This can be restated as

$$
\frac{s^{\prime}(p)-s^{\prime}\left(p_{k}\right)}{s(p)-s\left(p_{k}\right)}
$$

increasing or decreasing in $p$ (for all $p>p_{k}$ ). The derivative of (21) with respect to $p$ has the sign of:

$$
\begin{aligned}
& s^{\prime \prime}(p)\left(s(p)-s\left(p_{k}\right)\right)-s^{\prime}(p)\left(s^{\prime}(p)-s^{\prime}\left(p_{k}\right)\right) \\
= & s^{\prime \prime}(p) \int_{p_{k}}^{p} s^{\prime}(x) d x-s^{\prime}(p) \int_{p_{k}}^{p}\left(s^{\prime \prime}(x)\right) d x
\end{aligned}
$$

which in turn has the sign of

$$
\frac{s^{\prime \prime}(p)}{s^{\prime}(p)}-\frac{\int_{p_{k}}^{p} \frac{\left(s^{\prime \prime}(x)\right)}{s^{\prime}(x)} s^{\prime}(x) d x}{\int_{p_{k}}^{p} s^{\prime}(x) d x} .
$$

The second term is a weighted average of the coefficient of absolute risk aversion of $s$ for values between $p_{k}$ and $p$. So if $s^{\prime \prime}(x) / s^{\prime}(x)$ is increasing (resp. decreasing) in $x$, then this difference will be positive (resp. negative).

For concave $s$ the signs in inequality (20) flip, as $s^{\prime}\left(p_{h+1}\right)-s^{\prime}\left(p_{k}\right)<0$,so the results will be reversed.

\section{Proof of Proposition 7}

Let $g(z)=\frac{1}{2}\left(z_{L}(z)+z_{H}(z)\right)$. The optimal threshold is a fixed point of this function. When $z \rightarrow z_{\max }$ (or as $z \rightarrow \infty$ in the case of unbounded support), $g(z) \rightarrow \frac{1}{2} \bar{z}+\frac{1}{2} z<z$, and when $z \rightarrow z_{\min }$ (or as $z \rightarrow-\infty$ in the case of unbounded support), $g(z) \rightarrow \frac{1}{2} z_{\min }+\frac{1}{2} \bar{z}>z$. Consider the case where $\bar{z}>z_{\text {median }}$, which is illustrated in Figure 3 . For $z=z_{\text {median }}, g(z)=\bar{z}>z$. Since the function $g(z)$ is increasing and continuous, the unique fixed point $z^{*}$ must be to the right of $z_{\text {median }}$ and, as a consequence, $z^{*}>\bar{z}$, as illustrated in the figure. The proof for the 


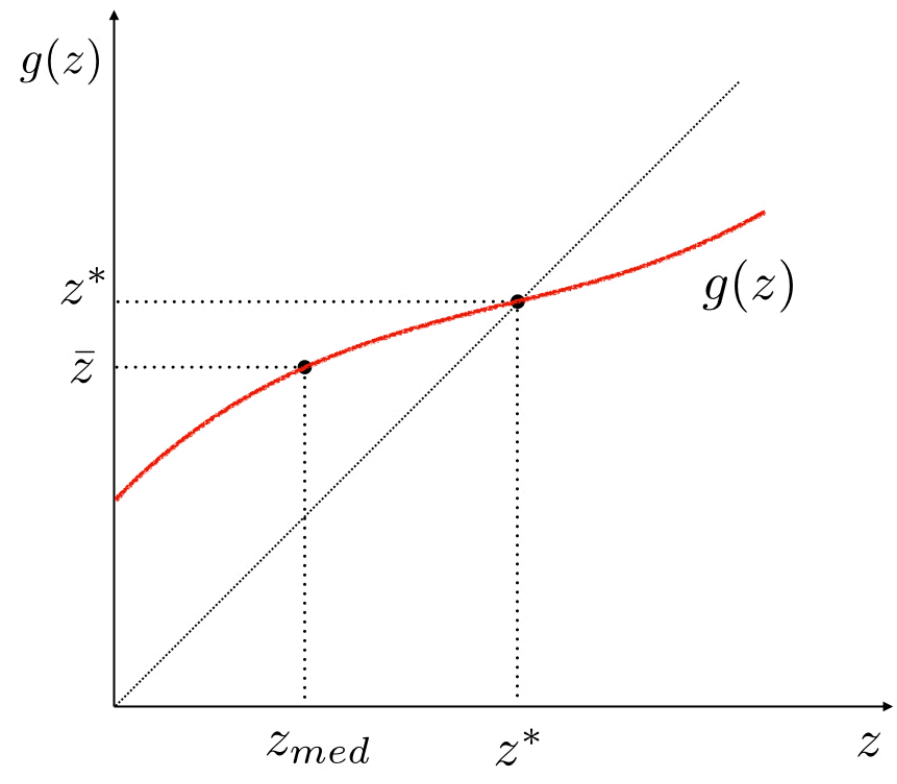

Figure 3: $z^{*}$ When Mean $>$ Median

case where $\bar{z}<z_{\text {median }}$ follows from a similar argument.

\section{Proof of Lemma 7}

Let

$$
\begin{aligned}
U\left(Q_{H}, Q_{L}, z^{*}\right)= & \max _{A_{L}, A_{H}} \int_{A_{L}} u\left(\theta, z_{L}\left(z^{*}\right)\right) d \Psi(\theta)+\int_{A_{H}} u\left(\theta, z_{H}\left(z^{*}\right)\right) d \Psi(\theta) \\
& \text { subject to: } \int_{A_{L}} d \Psi(\theta)=Q_{L} \text { and } \int_{A_{H}} d \Psi(\theta)=Q_{H}
\end{aligned}
$$

Given this we can write the general problem as

$$
V\left(z^{*}\right)=\max _{q_{L}, q_{H}} U\left(q_{L} F\left(z^{*}\right), q_{H}\left(1-F\left(z^{*}\right)\right), z^{*}\right)-F\left(z^{*}\right) c\left(q_{L}\right)-\left(1-F\left(z^{*}\right)\right) c\left(q_{H}\right)
$$

By the envelope theorem,

$$
\begin{aligned}
\frac{\partial V\left(z^{*}\right)}{\partial z^{*}} & =\frac{\partial U}{\partial Q_{L}} q_{L} f\left(z^{*}\right)-\frac{\partial U}{\partial Q_{H}} q_{H} f\left(z^{*}\right)+\frac{\partial U}{\partial z^{*}} \\
& -f\left(z^{*}\right) c\left(q_{L}\right)+f\left(z^{*}\right) c\left(q_{H}\right)
\end{aligned}
$$


The first two derivatives are the respective multipliers $p_{L}$ and $p_{H}$ of the constraints in (22). To evaluate the last term, first we note that:

$$
\frac{\partial z_{L}}{\partial z^{*}}=f\left(z^{*}\right) \frac{z^{*}-z_{L}\left(z^{*}\right)}{F\left(z^{*}\right)}
$$

and

$$
\frac{\partial z_{H}}{\partial z^{*}}=f\left(z^{*}\right) \frac{z_{H}\left(z^{*}\right)-z^{*}}{1-F\left(z^{*}\right)}
$$

Note also that:

$$
\begin{aligned}
\frac{\partial U}{\partial z^{*}} & =\left(\int_{\left(\theta_{1}, \theta_{0}\right) \epsilon A_{L}} \theta_{1} d G\right)\left(\frac{\partial z_{L}}{\partial z^{*}}\right) \\
& +\left(\int_{\left(\theta_{1}, \theta_{0}\right) \epsilon A_{H}} \theta_{1} d G\right)\left(\frac{\partial z_{H}}{\partial z^{*}}\right)
\end{aligned}
$$

Finally note that the measure of the set $A_{L}$ is $Q_{L}=q_{L} F\left(z^{*}\right)$ and the measure of the set $A_{H}$ is $Q_{H}=q_{H}\left(1-F\left(z^{*}\right)\right)$. Dividing and multiplying (26) by this respective measures and substituting (24) and (25) we get:

$$
\begin{aligned}
\frac{\partial U}{\partial z^{*}} & =f\left(z^{*}\right)\left(z^{*}-z_{L}\right) E\left(\theta_{1} \mid\left(\theta_{0}, \theta_{1}\right) \epsilon A_{L}\right) q_{L} \\
& +f\left(z^{*}\right)\left(z_{H}-z^{*}\right) E\left(\theta_{1} \mid\left(\theta_{0}, \theta_{1}\right) \epsilon A_{H}\right) q_{H}
\end{aligned}
$$

Substituting (27) in (23) we obtain:

$$
\begin{aligned}
\frac{1}{f\left(z^{*}\right)} \frac{\partial V}{\partial z^{*}} & =p_{L} q_{L}-c\left(q_{L}\right)-\left[p_{H} q_{H}-c\left(q_{H}\right)\right] \\
& +\left(z^{*}-z_{L}\right) E\left(\theta_{1} \mid\left(\theta_{0}, \theta_{1}\right) \epsilon A_{L}\right) q_{L} \\
& +\left(z_{H}-z^{*}\right) E\left(\theta_{1} \mid\left(\theta_{0}, \theta_{1}\right) \epsilon A_{H}\right) q_{H}
\end{aligned}
$$

Noting that the multipliers $p_{L}$ and $p_{H}$ are also the equilibrium prices, we can rewrite the first order condition for the optimal $z^{*}$ as:

$$
\begin{aligned}
\Pi\left(p_{H}\right)-\Pi\left(p_{L}\right) & =\left(z^{*}-z_{L}\right) E\left(\theta_{1} \mid\left(\theta_{0}, \theta_{1}\right) \epsilon A_{L}\right) q_{L} \\
& +\left(z_{H}-z^{*}\right) E\left(\theta_{1} \mid\left(\theta_{0}, \theta_{1}\right) \epsilon A_{H}\right) q_{H}
\end{aligned}
$$

